\title{
Severe nondiabetic plantar mal perforant in an adolescent girl with anorexia nervosa
}

\author{
C. Rezakhany' ${ }^{1}$ S. B. Guessoum ${ }^{1,2,3} \cdot$ M. R. Moro ${ }^{1,2,3} \cdot$ C. Blanchet $^{1,2,3}$
}

Received: 14 March 2021 / Accepted: 17 June 2021 / Published online: 24 June 2021

(c) The Author(s), under exclusive licence to Springer Nature Switzerland AG 2021

\begin{abstract}
Purpose We describe the case of a severe skin ulcer of the heel in an adolescent girl hospitalised for anorexia nervosa (AN) associated with a severe excessive exercise.

Methods This case report includes retrospective anamnestic and clinical data from the patient's medical record. A literature review was conducted on the dermatological changes described in AN.

Conclusion Severe starving syndrome in AN due to intake restriction and pathological associated behaviours can cause serious somatic complications, including psycho-dermatological lesions. We describe a rare case of a nondiabetic plantar "mal perforant" resulting from multiple risk factors including prolonged undernutrition, excessive exercise, and significant psychiatric comorbidity with self-injury. We suggest that, in this context, one should include a particular attention to the risk of non-visible skin complications reflecting both the AN symptoms severity and the severity of the patient's psychological distress requiring individualised and specialised multidisciplinary approach.
\end{abstract}

Level of evidence Level $\mathrm{V}$, case report

Keywords Anorexia nervosa $\cdot$ Adolescent $\cdot$ Skin complications $\cdot$ Self-injury $\cdot$ Excessive exercise $\cdot$ Psycho-dermatology $\cdot$ Psychiatric comorbidity

\section{Introduction}

Eating disorders are complex and multifactorial psychiatric disorders with frequent, acute or chronic, nutritional and somatic repercussions. In anorexia nervosa (AN), the most represented type of eating disorder in adolescents, undernutrition can lead to a multitude of organic complications including dermatological ones. Classically, the most common skin and appendages modifications described in AN are xerosis, lanugo-like body hair, carotenoderma, hyperpigmentation, pressure sores, alopecia, Russel's sign, paronychia, acrocyanosis and acral coldness [1]. Surprisingly, self-harmed skin lesions are often forgotten in this list;

C. Blanchet

corinne.blanchet@aphp.fr

1 APHP, Hôpital Cochin, Maison de Solenn, 75014 Paris, France

2 Université de Paris, PCPP, 92100 Boulogne-Billancourt, France

3 Université Paris-Saclay, UVSQ, Inserm, CESP, Team DevPsy, 94807 Villejuif, France whereas, many patients with eating disorders show a high prevalence of self-injurious events used as emotion-regulation behaviours [2]. Non-suicidal self-injury is observed in about $20 \%$ of patients with AN and can be another cause of skin lesions including scarifications and self-mutilations [3]. The self-injurious behaviours are numerous and include cutting, hitting and scratching. Most often, arms and hands are affected, but lesions on abdomen, legs or face are also reported. Excessive exercise, another kind of body selfabuse, can be a severe comorbidity of $\mathrm{AN}$ and has a wide prevalence, ranging from 31 to $80 \%$ in the literature [4, 4]. Besides the starving syndrome, high-intensity exercise can induce or worsen the somatic and physical complications associated with AN. Nevertheless, recommendations for medical examination, and somatic follow-up in AN are highly variable in the literature. We describe the case of an adolescent girl with AN and excessive exercise, presenting a severe skin ulcer of the heel. 


\section{Case presentation}

A 16-year-old girl was admitted for a severe restrictive type of AN, in the eating disorder department of a specialised medical unit for adolescents. The patient had no previous medical somatic history, her maximum weight before the anorexia onset was $47 \mathrm{~kg}$ for $1.61 \mathrm{~m}$ height at the age of 14 years (BMI $18 \mathrm{~kg} / \mathrm{m}^{2}$ ). Psychiatric history included obsessive-compulsive disorder since childhood with checking behaviours, which had been treated with a few sophrology sessions. Dietary restrictions and anorexic cognitions began two years prior to the admission. Major problematic exercise appeared at the same time and took a growing place in our patient's symptomatology. Initially, it consisted in staying up for several hours with an inability to sit and rest, to control shape and weight. Few months after the disease onset, her menstrual bleeding stopped. The patient developed at the same time anxiety and depressive symptoms including lack of concentration, loss of energy, apathy, sadness and social withdrawal. The patient showed growing concern in relation to food intake: she developed fat phobia with obsessive thoughts about losing weight, along with a disturbed body image. Her family noticed significant weight loss and a change in eating behaviours. She had compulsive rituals, such as cutting food into small pieces, eating slowly, arranging food in her plate in a certain order, and calorie counting. Furthermore, obsessive-compulsive disorder, excessive exercise and self-injurious behaviours worsened: she walked for hours along walls at home, hitting herself on corners, and self-mutilating with scratching and cutting. In this context, she began an ambulatory follow-up with multidisciplinary care including nutritional and psychiatric treatment with individual psychotherapy. Despite a regular and intensive treatment, her medical condition worsened with a profound weight loss $(12 \mathrm{~kg}$ ), leading to a first hospitalisation in a paediatric unit for 3 months (admission weight $35 \mathrm{~kg}$, height $1.63 \mathrm{~m}$, BMI $13 \mathrm{~kg} / \mathrm{m}^{2}$ ). A nasogastric tube feeding had to be used because of a total aphagia. An antidepressant (Sertraline $75 \mathrm{mg} /$ day) plus low-dose benzodiazepine (Diazepam) were started because of worsening of her depressive and anxiety symptoms. After discharge (weight $43 \mathrm{~kg}$, height $1.63 \mathrm{~m}$, BMI $16.18 \mathrm{~kg} / \mathrm{m}^{2}$ ), the patient began a day hospitalisation ( 5 days per week) in an eating disorders psychiatric unit. One month later, after a decline in her mental state and in her global clinical condition, with an accelerating weight loss, she was admitted for full-time hospitalisation in our adolescent medical unit (admission weight $40 \mathrm{~kg}$, height $1.63 \mathrm{~m}$, BMI $15.05 \mathrm{~kg} / \mathrm{m}^{2}$ ). This hospitalisation lasted for 6 weeks, during which she received intensive high-calorie refeeding, participated in individual psychological interviews, family therapy sessions, and group therapy with other patients. Weight gain was satisfying (weight $43.8 \mathrm{~kg}$, height $1.63 \mathrm{~m}$, BMI $16.4 \mathrm{~kg} / \mathrm{m}^{2}$ ) and mood improved, allowing hospital discharge. During the two following months, despite appropriate somatic and psychiatric ambulatory care, the medical condition worsened again. She had major food restriction close to aphagia, and severe psychiatric symptoms. Indeed, she presented permanent and massive anxiety about gaining weight, signs of depression and low self-esteem, self-injurious behaviours, marked obsessive-compulsive disorder, and major physical hyperactivity. In the context of many closures and restrictions in ambulatory care units due to the COVID-19 pandemic, added to parental exhaustion, the patient was admitted for a third hospitalisation in our adolescent inpatient medical unit. On admission, BMI was $15.4 \mathrm{~kg} / \mathrm{m}^{2}(41 \mathrm{~kg} / 1.63 \mathrm{~m})$, exclusive enteral nutrition by nasogastric tube was started at $400 \mathrm{kcal} / \mathrm{d}$ (around $10 \mathrm{kcal} /$ $\mathrm{kg} / \mathrm{d}$ ), with an initial increase of 300-400 kcal per week to $1500 \mathrm{kcal} / \mathrm{d}$. We evaluated her vitamin D level which was severely low, so vitamin D supplementation was started during the hospitalisation. We also began polyvitamin hydrosol, phosphorus and zinc oligosol supplementation to prevent refeeding syndrome. A bone densitometry using dual-energy X-ray absorptiometry (DEXA) showed a low bone mineral density (lumbar spine: $-2 \mathrm{Z}$ score, hip: $-1.5 \mathrm{Z}$ score). Food introduction was challenging because of the patient's difficulty in accepting weight modifications. She still presented severe anxiety, insomnia, and severe self-injurious behaviours, such as hitting and cutting herself. Physical hyperactivity increased considerably, as a compensatory behaviour to prevent weight gain induced by enteral nutrition but also as a strategy to fight anxiety. She was unable to sit, walked during hours (more than five consecutive hours) in the halls, refused to participate in therapeutic activities because she could not sit, and did not enter into social relations with other patients. Systematic physical examinations were done every week by a paediatrician, and blood tests were regularly practiced. An individual and family psychiatric approach was initiated at admission. Major depressive disorder was confirmed, and an antidepressant treatment was pursued (Sertraline $100 \mathrm{mg}$ ). We continued benzodiazepines (Diazepam $5 \mathrm{mg}$ ) and started Cyamemazine $20 \mathrm{mg}$ (first generation antipsychotic treatment usually used as a brief adjunctive treatment for anxiety and insomnia). In the fourth month of hospitalisation, a systematic physical examination found a severe feet hyperkeratosis, and horn erosions of the left heel were diagnosed. The lesions seemed completely painless, with no complaints from the patient who had not noticed them. Two days later, the lesions worsened into inflammatory blisters aggravated by self-mutilation, leading to an ulcerative wound of $10 \mathrm{~cm} \times 3 \mathrm{~cm}$. There were inflammatory signs surrounded by a border of thickened 


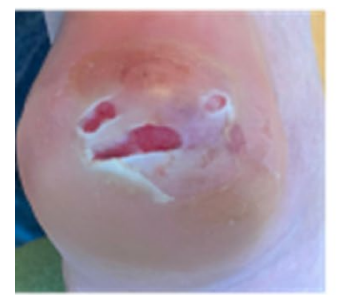

(a) day 1

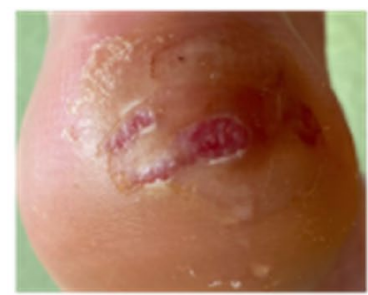

(b) day 15

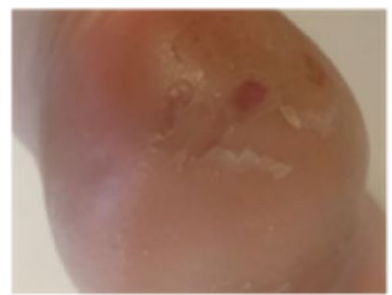

(c) day 30

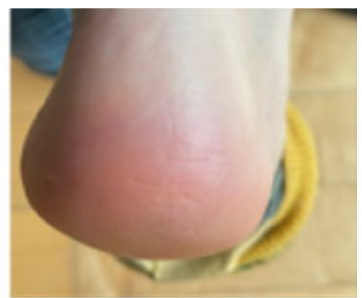

(d) month 3

Fig. 1 Description of the evolution of a skin ulcer in an adolescent patient with AN from day one to month three

skin (Fig. 1a). Specialised dermatological advice was sought to deal with this plantar ulcer. We initiated a daily Jelonet bandage, and we prescribed a «Barouk botte», an orthopaedic shoe which reduces the pressure on the heel. During the wound healing period, we tried to increase enteral refeeding to restore an adapted nutritional status, but the patient's increasing psychological difficulties, fear of gaining weight and the worsening of problematic physical exercise did not allow weight normalisation. During a month, a medical examination was done two times a week, along with a nursing daily local care. Psychiatric symptoms did not improve; thus, Sertraline was switched to Venlafaxine LP $150 \mathrm{mg}$, and later $225 \mathrm{mg}$. Cyamemazine and Diazepam doses were also increased. The patient began hypnosis and psychomotor therapy sessions. As the weeks went by, a progressive re-epithelialisation (Fig. 1b, Fig. 1c) occurred with complete wound healing. Further dermatological evaluations were satisfying, and there was no recurrence three months later (Fig. 1d).

\section{Discussion}

To our knowledge, this is the first description of a heel lesion, we could call a nondiabetic plantar "mal perforant", in a patient suffering from AN. Multiple deficiencies resulting from restrictive dietary intakes and weight loss are widely described in undernutritional states in patients with eating disorders. Such deficiencies may concern many factors with key roles in skin renewal, collagen production, and skin elasticity (vitamin C, zinc, iron). This leads to trophic disorders and skin atrophy that can partly explain the skin fragility and the occurrence of pressure sores [6]. Indeed, our patient showed signs of skin fragility with bruises and wounds after minor traumas. Furthermore, AN is associated with multiple and profound endocrine disturbances as a result of disturbed regulation of adrenal, gonadal, thyroid, and somatotropic hypothalamic pituitary axes. These disturbances are known to be associated with altered peripheral endocrine metabolism. Chronic hypercorticism, in response to undernutrition-induced stress, has also been observed, and can induce muscular atrophy and cutaneous fragility [7]. There is also a dysregulation between peripheral vasoconstriction and vasodilatation leading to peripheral vascular abnormalities. It is called acrosyndrome, and it has also already been reported in the literature. This may explain the capillary fragility of these patients and the high risk of developing vascular wounds [8]. Among neurological complications, previous studies have reported an increased risk of developing localised compression neuropathies secondary to subcutaneous tissue loss. Moreover, peripheral neuropathy has also been described, particularly in long-standing cases of AN, which is most likely a product of chronic starvation rather than a specific nutrient deficiency [9]. In addition to the modifications due to undernutrition, a reduced perception of pain is a well-established phenomenon in patients with AN. Some studies indicating elevated pain threshold associated with an increased parasympathetic tone and a low-T3 syndrome [10]. Furthermore, these patients can have modifications in their body perception. Especially, structural and functional brain abnormalities are reported in regions involved in perception and body stimuli integration [11]. This reduced perception of pain, due to peripheral neuropathy and modifications in body perception, probably played a key role in the delayed diagnosis of the lesion. These findings highlight the necessity of periodic and frequent medical examination. However, most patients with AN do not show such severe and unusual dermatological complications. We assumed that psychiatric factors such as hyperactivity, self-injurious behaviours, and dermatillomania, may have contributed to worsen this skin lesion. Self-mutilation can be regarded as the expression of an intense psychological pain through body self-injury. High-intensity exercise, a frequent symptom of AN, interferes with nutrition rehabilitation and increases the risk of short-term somatic complications [12] [13]. Our patient exhibited severe problematic exercise since the AN onset, which worsened during the refeeding as a compensatory behaviour to prevent weight gain. Prolonged standing was certainly involved in her skin lesion onset and worsening. In the case of our patient, the self-injurious behaviour began in the early adolescence, and continued during hospitalisation. Furthermore, she admitted self-manipulation of her skin 
lesion, as a form of psychogenic excoriations. Dermatillomania, the frequency of which is high in patients suffering from AN, is related to obsessive-compulsive disorder according to DSM-5. These psychiatric symptoms certainly impacted the onset and persistence of the skin lesion, but they also interfered with the treatment. Cognitive rigidity, persistent problematic physical activity, massive anorexic cognitions and resistance to care observed in this patient have been an obstacle to optimised refeeding and care, as frequently reported in AN [14]. Indeed, although dermatologists prescribed rest and offloading interventions to reduce the plantar pressure, our patient was unable to rest and sit because of her excessive exercise. In this context, we had to prescribe an orthopedic shoe for pressure offload on her heel while she continued to walk. Moreover, this kind of skin lesion may be at high risk of bacterial superinfection because of an immune dysregulation associated with AN, particularly because of neutropenia (16.3\%), which may result in lifethreatening septicemia [15]. Thus, we assume that dermatological lesions of the feet in severe AN, which are probably of multifactorial origin, are poorly known, under-diagnosed with no standardised treatment. As recently reported, Trifarotene, a new fourth-generation retinoid with a reduced toxicity and an increased efficacy, could have been proposed in the absence of a favourable outcome for this kind of ulceration of unclear etiology. It is indeed known that vitamin A and its synthetic analogues have a crucial role in modulating some skin functions as epidermal keratinization, differentiation, maturation, and proliferation [16]. We suggest that changes in body perception, decreased pain sensibility, denial of the disorder and self-harm behaviours associated with psychiatric comorbidities may prevent patient reporting of skin lesions and, thus, may delay diagnosis. The unusual nature and the extreme severity of this nondiabetic plantar "mal perforant" are the reflection of both the chronic undernutrition state and the intensity of the patient psychological distress. Based on this case of psycho-dermatological lesion, we suggest that rigorous and careful monitoring of the skin condition should be an indispensable part of the medical and nutritional integrated care in a multidisciplinary approach in patients suffering from $\mathrm{AN}$, especially for those with severe psychiatric comorbidity.

\section{Strength and limits}

This case reports an unrecognised and probably underdiagnosed complication in AN. Further studies investigating prevalence and risk factors and proposing care recommendations might be of interest.

\section{What is already known on this subject?}

Self-harm skin lesions are frequent in AN and connected to adolescent psychological distress. While excessive exercise is a well-known comorbidity, skin feet lesions are poorly described in AN.

\section{What does this study add?}

Severe skin foot wounds in AN adolescent should be known to care givers and detected early, particularly in a context of complex comorbidities. Multidisciplinary treatment strategy is also discussed.

Author contributions Conceptualization: $\mathrm{CR}, \mathrm{CB}$; Validation: $\mathrm{CR}$, SBG, MRM, CB; Drafting: CR, CB; Review and Editing: CR, SBG, MRM, CB.

Funding None.

Availability of data and materials All data and material are available.

\section{Declarations}

Conflict of interest On behalf of all authors, the corresponding author states that there is no conflict of interest.

Compliance with ethical standards Authors have checked that they are complying with the specific and ethical requirements of their institution.

Informed consent Written informed consent for publication of the clinical details and clinical images was obtained from the parents of the patient. A copy of the consent form is available for review by the Editor of Eating and weight disorders Journal.

\section{References}

1. Strumia R (2009) Skin signs in anorexia nervosa. Dermatoendocrinology 1:268-270. https://doi.org/10.1159/000051779

2. Smithuis L, Kool-Goudzwaard N, Janneke M et al (2018) Selfinjurious behaviour in patients with anorexia nervosa: a quantitative study. J Eat Disord 6:1-10. https://doi.org/10.1186/ s40337-018-0214-2

3. Davico C, Amianto F, Gaiotti F et al (2019) Clinical and personality characteristics of adolescents with anorexia nervosa with or without non-suicidal self-injurious behavior. Compr Psychiatry 94:152115. https://doi.org/10.1016/j.comppsych. 2019.152115

4. Rizk M, Lalanne C, Berthoz S et al (2015) Problematic exercise in anorexia nervosa: testing potential risk factors against different definitions. PLoS ONE 10:e0143352. https://doi.org/10. 1371/journal.pone.0143352 
5. Mond J, Gorrell S (2021) "Excessive exercise" in eating disorders research: problems of definition and perspective. Springer, Berlin. https://doi.org/10.1007/s40519-020-01075-3

6. Shibuya S, Ozawa Y, Toda T et al (2014) Collagen peptide and vitamin $\mathrm{C}$ additively attenuate age-related skin atrophy in Sod1deficient mice. Biosci Biotechnol Biochem 78:1212-1220. https:// doi.org/10.1080/09168451.2014.915728

7. Gosseaume C, Dicembre M, Bemer P et al (2019) Somatic complications and nutritional management of anorexia nervosa. Clin Nutr Exp 28:2-10. https://doi.org/10.1016/j.yclnex.2019.09.001

8. De Martinis M, Sirufo MM, Ginaldi L (2018) Raynaud's phenomenon and nailfold capillaroscopic findings in anorexia nervosa. Curr Med Res Opin 34:547-550. https://doi.org/10.1080/03007 995.2017.1417828

9. Renthal W, Marin-Valencia I, Evans PA (2014) Thiamine deficiency secondary to anorexia nervosa: an uncommon cause of peripheral neuropathy and Wernicke encephalopathy in adolescence. Pediatr Neurol 51:100-103. https://doi.org/10.1016/j.pedia trneurol.2014.03.025

10. Bär K-J, Boettger S, Wagner G et al (2006) Changes of pain perception, autonomic function, and endocrine parameters during treatment of anorectic adolescents. J Am Acad Child Adolesc Psychiatry 45:1068-1076. https://doi.org/10.1097/01.chi.00002 27876.19909.48

11. Bär K-J, de la Cruz F, Berger S et al (2015) Structural and functional differences in the cingulate cortex relate to disease severity in anorexia nervosa. J Psychiatry Neurosci 40:269-279. https:// doi.org/10.1503/jpn.14019

12. Rizk M, Kern L, Lalanne C et al (2019) High-intensity exercise is associated with a better nutritional status in anorexia nervosa. Eur Eat Disord Rev 27:391-400. https://doi.org/10.1002/erv.2661

13. Blanchet-Collet C, Sider A, Gal B et al (2016) Anorexia nervosa hyperactivity-induced ischemic colitis (ANHIC): a new cause of anaemia. Eat Weight Disord Stud Anorexia Bulimia Obes 21:507510. https://doi.org/10.1007/s40519-016-0295-3

14. Sheynblyum $M(2019)$ Cognitive rigidity in patients with anorexia nervosa. The Science Journal of the Lander College of Arts and Sciences 13:10. https://touroscholar.touro.edu/sjlcas/vol13/iss1/ 10

15. Ibrahim N, Barruchet A, Moro MR, Blanchet C (2020) Severe neutropenia in an anorexic adolescent girl: a stigma of underfeeding syndrome? Eat Weight Disord. https://doi.org/10.1007/ s40519-020-01016-0

16. Cosio T, Di Prete M, Gaziano R et al (2021) (2021) Trifarotene: a current review and perspectives in dermatology. Biomedicines 9:237. https://doi.org/10.3390/biomedicines9030237

Publisher's Note Springer Nature remains neutral with regard to jurisdictional claims in published maps and institutional affiliations. 